\title{
Post-buckling analysis of a simply supported compound beams made of two symmetrically distributed materials under uniform thermal loading
}

\author{
V. Sridhar Patnaik ${ }^{1}$, G. Venkateswara Rao ${ }^{2}$ and A.V.S.S.K.S. Gupta ${ }^{3}$ \\ Associate Professor, Department of Mechanical Engineering, VITAM college of Engineering, Visakhapatnam \\ - 531 173, India \\ Research Professor, Department of Mechanical Engineering, Vardhaman College of Engineering, Shamshabad \\ , Hyderabad-501 218, India. \\ Professor, Department of Mechanical Engineering, Jawaharlal Nehru Technological University, Hyderabad- \\ 500085 , India
}

\begin{abstract}
Thermal post buckling analysis of axi symmetric compound stepped beams, made of two different materials with axially immovable ends, for simply supported conditions, and was studied numerically. The analysis was performed by using the energy principle. The compound beam is made of two different materials, with the end segments of equal lengths of the same material with a higher, and the central segment of a different length of another material with lower coefficients of linear thermal expansion. Similarly, the Young's modulus of the material of the middle segment is higher than that of the symmetrically placed end segments. The buckling and post buckling strength of stepped compound beam is predicted by examining the influence of inertia, length and deflection ratios. As mentioned in the title of the present work, importance is given for predicting the non dimensional post buckling parameters for the two different material combinations such as steel-aluminum, titanium-aluminum and copper-aluminum as these combinations of materials finds a major applications in the fields of aerospace, electronic, power generation and automobile industry where high strength and low weight are desirable.

For post buckling analysis, the accuracy and efficiency of the present method for a isotropic uniform beam with simply supported and fixed-fixed boundary conditions, have shown a good agreement with that of obtained by finite element method.
\end{abstract}

Key words: compound beam, copper-aluminum, steel-aluminum, titanium-aluminum, and post buckling

\section{Introduction}

The emergence of large variety of structural members made of two different materials, in the interest of restoration and economy, has inspired researchers to evaluate the contribution of the engineering materials to the structural strength at elevated temperatures. This study has been undertaken for the purpose of identifying the behavior of stepped beams, made of two different materials such as steel-aluminum, titanium-aluminum and copper-aluminum. The previous analytical and numerical studies on the post buckling behavior of isotropic uniform beams for simply supported and fixed-fixed end conditions are used to confirm the validity of results obtained by present method for different materials.

Fundamental principles of structural members under the influence of thermal and mechanical loads are reported in the work of Usmani et al [1]. The fundamental principles presented in this paper provide a means of estimating forces and displacements in real structures with appropriate idealizations.Raju and Rao [2-6] have studied the thermal post buckling of uniform and tapered columns with immovable ends, using Rayleigh-Ritz and the finite element methods. In this study an exact mathematical model thermal post buckling behavior of uniformly heated elastic rods has also been developed. Thermal post buckling behavior of heated elastic rods has also been predicted in this study. A simple intuitive method to predict the thermal post buckling behavior of uniform columns has been presented by Rao and Raju. Different types of boundary conditions for the columns have been presented in this study. Li and Batra [7] employed a numerical method, such as shooting method, in the study and analysis of deformations of homogenous and isotropic Euler-Bernoulli beams with simply supported and fixed-fixed end conditions, heated uniformly into the post buckling regime.

The concept of a stepped compound beam is introduced in the present authors' research work $[8,9]$ to obtain a near optimum (minimum mass) configuration with respect to critical buckling temperature of the stepped symmetric compound beam for different boundary conditions and different material combinations. The nonlinear ordinary differential equations deals with post buckling behavior of beams subject to compressive loads have analyzed either numerically or analytically in the work of [10-12].Jeket [13] has studied deformations of a thermally buckled beam made of nonlinear thermo elastic material. 
Sherbourne,A.N. [14] and Pandy and Sherbourne[15] have reported the buckling and post buckling of composite beams and plates by using differential quadrature multiplier principle, DQM. Li and Balachandran [16] have analyzed the thermal buckling and free vibrations of composite micro electromechanical resonators modeled as stepped composite Euler-Bernoulli beam. Thermal buckling strength of axially restrained composite structure is investigated by Kardomates et al [17]. The pinned-fixed beam resting on elastic foundation is investigated by Xisong and Shimrong Li [18].Post buckling behavior of columns subjected to mechanical loads was discussed by Dym [19] and Thompson and Hunt [20] using differential approach.Buckling and vibration analysis of beams and plates for a wide range of anisotropic materials are addressed by Laszlo P. kollar and George S.Springer [22].

Postbuckling deformations of perfect and geometrically imperfect columns resting on elastic foundations have been analysed by Koundis et al [23]. It was observed that the critical state of a perfect column is a stable symmetric bifurcation point. One of the major problems faced in realizing the compound beams is the joining of different materials efficiently and effectively performed by the modern techniques, especially friction welding, friction stir welding developed by the researchers reported in Refs. [24-25].

In the present work, energy principle is used to predict the thermal post buckling behavior of uniform and stepped beams with simply supported boundary conditions, made of single or two different materials combinations.

\subsection{Evaluation of Thermal Buckling Load}

\section{Mathematical Formulation}

In this present method, concept leads only one single undetermined constant which can be solved by the principle of conservation of total energy.

Expressions for strain energy $\mathrm{U}$, and potential energy $\mathrm{W}$ for the external applied compressive load $\mathrm{P}$ (E $\alpha \mathrm{TA}$ ), caused due to the temperature rise, for the right half of the three element symmetric beam, made of single material as shown in Fig.la or two different materials ,known as compound beam as shown in Fig.1b $\mathrm{Y}(\mathrm{L} / 2)=0$, during buckling, are given by

$U=\int_{0}^{L_{2} / 2} \frac{M^{2}}{2 E_{2} I_{2}} d x+\int_{L_{2} / 2}^{L / 2} \frac{M^{2}}{2 E_{1} I_{1}} d x$

$W=\frac{P}{2} \int_{0}^{L_{2} / 2}\left(\frac{d y}{d x}\right)^{2} d x+\frac{P}{2} \int_{L_{2} / 2}^{L / 2}\left(\frac{d y}{d x}\right)^{2} d x$

Where EI is the flexural rigidity and $\mathrm{L}$ is the full length of the three segment symmetric beam.

Now from the principle of minimization of total potential energy, we have,

$\partial(U-W)=0$

For the case of simply supported beam with immovable ends, the admissible function for the lateral displacement ' $Y$ ' that satisfy the geometric boundary conditions, considering the right half of the beam because of symmetry, as shown in Fig.1a, $\mathrm{Y}(\mathrm{L} / 2)=0$, in non-dimensional form is given by

$Y=a\left[1-4.8\left(\frac{x}{L}\right)^{2}+3.2\left(\frac{x}{L}\right)^{4}\right]$

Substituting the expressions for $\mathrm{U}$ and $\mathrm{W}$ in eqn.3, gives the mechanical buckling load parameter, in nondimensional form as

$\lambda_{L M}=\frac{\mathrm{PL}^{2}}{\mathrm{E}_{2} \mathrm{I}_{2}}=\frac{2.486511477}{\left[\left(\frac{0.251936286}{\mathrm{E}_{12} \mathrm{I}_{12}}\right)+\left(0.0022 \zeta^{9}-0.0342857 \zeta^{7}+0.184 \zeta^{5}-0.4 \zeta^{3}+0.5 \zeta\right)\left(1-\left(\frac{1}{\mathrm{E}_{12} \mathrm{I}_{12}}\right)\right)\right]}$

The magnitude of restraining force $\mathrm{P}$, caused due to temperature rise $\mathrm{T}$ in the beam,

$P=\frac{A_{2} E_{2} \alpha_{2} T\left[(1-\zeta) \alpha_{12}+\zeta\right]}{\left[\left(\frac{1-\zeta}{E_{12} A_{12}}\right)+\zeta\right]}$

(6)

The equivalent thermal buckling load parameter in non-dimensional form $\lambda_{L T}$, is worked out from equations (5) \& (6) gives

$\lambda_{L T}=\frac{\mathrm{A}_{2} \alpha_{2} \mathrm{TL}^{2}}{\mathrm{I}_{2}}=\frac{\left[\left(\frac{1-\zeta}{\mathrm{E}_{12} \mathrm{~A} 12}\right)+\zeta\right]}{\left[(1-\zeta) \alpha_{12}+\zeta\right]}\left\{\frac{2.486511477}{\left[\left(\frac{0.251936286}{\mathrm{E}_{12} \mathrm{I}_{12}}\right)+\left(0.0022 \zeta^{9}-0.0342857 \zeta^{7}+0.184 \zeta^{5}-0.4 \zeta^{3}+0.5 \zeta\right)\left(1-\left(\frac{1}{\mathrm{E}_{12} \mathrm{I}_{12}}\right)\right)\right]}\right\}$

2.2 Evaluation of tension ' $T_{a}$ '

As the importance given in this presentation is to the stepped three segment symmetric beam made up of two different materials with immovable ends undergoes large lateral deformations, the axial tension developed , $T_{a}$, is given by 
$T_{a}=\frac{E_{2} I_{2}}{r_{2}^{2} L_{2}} \int_{0}^{L_{2} / 2}\left(\frac{d y}{d x}\right)^{2} d x+\frac{E_{1} I_{1}}{r_{1}^{2} L_{1}} \int_{L_{2} / 2}^{L / 2}\left(\frac{d y}{d x}\right)^{2} d x$

(8)

And in the non dimensional form is given by

$\lambda_{\mathrm{T}_{\mathrm{a}}}=\frac{\mathrm{T}_{\mathrm{a}} \mathrm{L}^{2}}{\mathrm{E}_{2} \mathrm{I}_{2}}=\frac{\mathrm{a}^{2}}{\mathrm{r}_{2}{ }^{2}}\left[\left(\frac{2.486511477 \mathrm{E}_{12} \mathrm{I}_{12}}{\mathrm{r}_{12}{ }^{2}(1-\zeta)}\right)+\left(0.182857 \zeta^{7}-1.536 \zeta^{5}+3.84 \zeta^{3}-0.000345523\right)\left(\frac{1}{\zeta}-\frac{\mathrm{E}_{12} \mathrm{I}_{12}}{\mathrm{r}_{12}{ }^{2}(1-\zeta)}\right)\right]$

(9)

The compressive force developed in the column, due to temperature raises $\mathrm{T}$ from the stress free condition under equilibrium conditions (linear elastic forces), reaches a critical value, known as the linear critical thermal $\operatorname{load} \lambda_{L T}$.

However, in the post buckling range, the compressive force developed also has to balance the axial tension developed due to large deformations in addition to the linear elastic forces. Thus the thermal post buckling load in the non-dimensional form is expressed as

$\lambda_{N L T}=\lambda_{L T}+\lambda_{\mathrm{T}_{\mathrm{a}}}$

(10)

Once the tension developed in the beam due to large deflections is known, the thermal post-buckling behavior of the column in terms of

$\frac{\lambda_{N L T}}{\lambda_{L T}}=1+\frac{\lambda_{T_{a}}}{\lambda_{L T}}$

(11)

Equation (11) gives the values of the ratio of thermal post buckling load parameter to buckling load parameter, $\frac{\lambda_{N L T}}{\lambda_{L T}}$ of a simply supported beam for specified values of ratios of $a / r_{2}, \zeta$ and $I_{12}$.

Apart from the theoretical analysis, a detailed Finite Element Analysis is also carried out to capture the thermal post-buckling path of stepped beam using commercially available software ANSYS. The stepped beam is discretized with 100 beam elements (BEAM 4 of ANSYS 11.0). Initial geometric imperfection of $1 / 100^{\text {th }}$ of beam radius is given at the middle length of the beam(line of symmetry). The thermal load is applied in temperature increments $(0.1 \mathrm{deg})$ from initial stress free temperature. Newton-Raphson procedure is used to solve the non-linear finite element system of equations. The analysis is carried out for the beam simply supported at both ends: translational dof at two ends are zero

\section{Numerical Results And Discussion}

The material properties, namely, the Young's modulus, density and linear coefficient of thermal expansion of aluminum, copper, steel and titanium, considered in this work are presented in Table 1 . Tables 2 to 7 show the values of the ratios of the thermal post buckling load to thermal buckling load in the non dimensional form $\left(\frac{\lambda_{N L T}}{\lambda_{L T}}\right)$ for the isotropic simply supported boundary conditions of uniform and stepped beams, obtained from the present theoretical analysis using energy principle and finite element analysis through software ANSYS for several values of central deflection parameter $a / r_{2}$, length ratio, $\zeta$ and moment of inertia ratio, $I_{12}$.

The maximum difference in results of present theoretical analysis shown outside the bracket and ANSYS, values shown inside the bracket, is around 4\%. The values of these ratios are decreasing with the increasing of length ratio and increasing with the increase of central deflection ratio and moment of inertia ratio.

Also it is observed from the table 2 that the present theoretical analysis and ANSYS values for a uniform simply supported and isotropic beam have shown good agreement with a difference of $0.001 \%$ and $4 \%$ with that of from Ref. [5]. Beyond this value of the difference goes on increasing with increasing of $\mathrm{a} / \mathrm{r}_{2}$ and a maximum of $0.535 \%$ for $\mathrm{a} / \mathrm{r}_{2}=3$. This difference is not very high for all practical purposes.

Tables 8 to 12 presenting the values of the ratios of the thermal post buckling load to thermal buckling load in the non dimensional form $\left(\frac{\lambda_{N L T}}{\lambda_{L T}}\right)$ for the simply supported boundary conditions of stepped compound beams, obtained from the present theoretical analysis using energy principle and finite element analysis through software ANSYS for several values of central deflection parameter $a / r_{2}$, length ratio, $\zeta$ and moment of inertia ratio, $I_{12}$. The results have shown the same trend as it is seen in results of the isotropic beam. Also observed that these values are higher for the material combination of titanium and aluminum due to larger value of thermal expansion ratio when compared with that from other material combinations of copper-aluminum and steelaluminum. It has been observed the ratios of $\left(\frac{\lambda_{N L T}}{\lambda_{L T}}\right)$ for the compound beams are lower than that of isotropic beams at higher values of length ratio but more significant at lower values of length ratio. This can be clearly observed in the compound beam of titanium-aluminum combination existed with larger value of thermal expansion ratio and moderate value of modulus ratio. The same authors already presented in the previous research publications [8-9], the effectiveness and advantage of a compound beam with respect to mass and critical buckling temperature. 


\section{Conclusions}

Thermal post buckling analysis of stepped,symmetric beams ,made of single material and combinations of different materials for simply supported boundary conditions with immovable ends, is investigated using energy principle by considering the dimensionless form of $4^{\text {th }}$ order algebraic function and by using ANSYS. Accuracy of these results for a compound beam is verified with those obtained from the finite element method through software ANSYS and can be seen a good agreement. The present study on post buckling of stepped compound beams serve as a reference for predicting the post buckling response of compound beam and its significance in applications.

\section{Acknowledgment}

The authors gratefully acknowledge the managements of the respective Institutes for their constant encouragement during the course of this work.

\section{References}

J.M. Usmani, Rotter, Lamont, S,.Sanad,A.M., and M.Gillie, "Fundamental Principles of Structural Behavior Under Thermal Effects", Fire Safety Jl. 2001, Vol. 36, pp 721-744.

G. V. Rao, and K. K. Raju , "Thermal Post Buckling of Columns,"AIAA Journal, Vol. 22, No. 6, 1984, pp. 850-851.

[3] G. V. Rao, , and K. K. Raju, ,"Thermal Post Buckling of Tapered Columns,” AIAA Journal, Vol. 22, No. 10, 1984, pp. 1499-1501.

[4] K. K. Raju, and G. V. Rao , "Thermal Post Buckling of Uniform Columns on Elastic Foundation," Journal of Engineering Mechanics,Vol. 119, No. 3, 1993, pp. 626-629.

[5] G. V. Rao, and K. K. Raju, "Thermal Post Buckling of Uniform Columns: A Simple Intuitive Method," AIAA Journal, Vol. 40, No. 10, 2002, pp. 2138-2140.

[6] G. V. Rao, and K. K. Raju, "Thermal Post Buckling of Uniform Columns on Elastic Foundation: Intuitive Solution," Journal of Engineering Mechanics, Vol. 129, No. 11, 2003, pp. 1351-1354.

[7] S.R.Li, and R.C. Batra, 'Thermal Buckling and Post buckling of Euler-Bernoulli beamsSupported on non linear elastic foundations', AIAA journal, vol.45, No.3, March 2007, pp.713-720.

[8] V.Sridhar Patnaik, G. V. Rao, , and A .V .S. S . Kumaraswamy Gupta, "Optimum configuration of stepped simply supported beam with respect to buckling temperature” journal of structural engineering, Vol. 39, No. 2, June-July 2012 pp. 178-183.

[9] V.Sridhar Patnaik, G. V. Rao, , and A .V .S. S . Kumaraswamy Gupta, "Near Optimum Configuration of Stepped Hinged Beams with Buckling Load Constraint" journal of structural engineering, Vol. 40, No. 2, June-July 2013 pp. 105-114

[10] T. Stemple, "Extensional Beam-Columns: An Exact Theory, "International Journal of Non-Linear Mechanics, Vol. 25, No. 6, 1990, pp. 615-623.

[11] C. Y. Wang, "Postbuckling of a Clamped-Simply Supported Elastica,"International Journal of Non-Linear Mechanics, Vol. 32, No. 6, 1997, pp. $1115-1122$.

[12] C. P.Filipich, and M. B. Rosales, "A Further Study on the Postbuckling of Extensible Elastic Rods," International Journal of NonLinear Mechanics, Vol. 35, No. 6, 2000, pp. 997-1022.

[13] T.Jekot, "Nonlinear Problems of Thermal Buckling of a Beam,"Journal of Thermal Stresses, Vol. 19, No. 4, 1996, pp. 359-369.

[14] A.N. Sherbourne, and M.D. Pandey, , (1991) Differential Quadrature Method in the Buckling Analysis of Beams and Composite Plates, Computers \& Structures, vol.40, pp.903-913.

[15] M.D. Pandey, and A.N. Sherbourne, (1991) Buckling of Anisotropic Composite Plates Under Stress Gradient,ASCE Journal of Engineering Mechanics, vol. 1 17, pp. 260-275.

[16] H.Li, and B. Balachandran, "Buckling and Free Oscillations of composite Micro resonators," Journal of Microelectromechanical Systems, Vol. 15, No. 1, 2005, pp. 42-51.

[17] L. Liu, G.A. Kardomateas, J.M Birman, Holmes, and G.J. Simitses, "Thermal Buckling, of a heat-exposed, axially restrained composite column" Fire behavior of Composites, Vol. 37, July 2006, pp 972-980.

[18] Xi Song and Shi-Rong Li, "Thermal buckling and post-buckling of pinned-fixed Euler-Bernoulli beams on an elastic foundation" Mech. Res. Communications, Vol. 34, No. 2, March 2007, pp 164-171.

[19] C.L. Dym, , "Stability theory and its applications to structural mechanics", NoordhoffInternational.Leyden, The Netherlands, 1974.

[20] J.M.T.Thompson, and G.W. Hunt , "A general theory of elastic stability, Wiley", London, 1973.

[21] S.P Timeshenko, and J.M. Gere, Theorey of elastic stability, McGraw Hill, New York,1961.

[22] P. Laszlo P. kollar and S. Springer George,"Mechanics of composite structures", CambridgeUniversity press

[23] A.N. Kounadis, J. Mallis, and A. Sbarounis, "Post buckling Analysis of Columns Resting On an Elastic Foundation," Archive of Applied Mechanics, Vol. 75, No. 6-7, 2006, pp. 395-404.

[24] E. Taban, J.E. Gould, and J.C Lippold,“ Dissimilar friction welding of 6061-T6 aluminum and AISI 1018 steel: Properties and micro structural characterization," Materials \& Design", Vol. 31, 2010, pp. 2305-2311.

[25] H. Seli, H. Izani, M. Ismail, E. Rachman, and Z.A.Ahmad, "Mechanical evaluation and thermal modeling of friction welding of mild steel and aluminum," Journal of Materials Processing Technology, Vol. 210, 2010, pp. 1209-1216.

\section{Notations:}

$\boldsymbol{L} \quad$ : Full length of the stepped symmetric beam

a : Central lateral deflection of the full length stepped symmetric beam.

$\boldsymbol{T} \quad$ : Temperature rise

$\boldsymbol{P} \quad$ : External applied compressive load $\left(A_{2} E_{2} \alpha_{2} T\right)$

$\boldsymbol{T}_{\boldsymbol{a}} \quad$ : Axial tension developed in the beam

$\boldsymbol{U} \quad$ : Strain energy as given by eqn. (1)

$\boldsymbol{W} \quad$ : work done by the thermal load due to temperature rise $T$

$\boldsymbol{Y} \quad$ : Lateral displacement 
$\boldsymbol{x} \quad$ :Axial coordinate

$\zeta \quad$ :Length ratio $x / L$,

$\boldsymbol{A}_{1}, \boldsymbol{A}_{2} \quad$ : Cross sectional areas of the elements 1 and 2 respectively

$\boldsymbol{L} / \mathbf{2} \quad$ : Length of the half section of the full length stepped symmetric beam

$L_{1} / 2, L_{2} / 2$ : Lengths of the half sections of the elements 1 and 2 of the Stepped symmetric beam respectively.

$\boldsymbol{E}_{1}, \boldsymbol{E}_{2}$ : Young's modulus of the elements 1 and 2 of the stepped symmetric circular beam respectively.

$\boldsymbol{\alpha}_{1}, \boldsymbol{\alpha}_{\mathbf{2}}$ : Linear coefficient of thermal expansions of the elements 1 and 2 of the stepped symmetric circular beam respectively.

$\boldsymbol{I}_{1}, \boldsymbol{I}_{2}$ : Areas moment of inertia of the elements 1 and 2 of the steppe symmetric circular beam respectively.

$\boldsymbol{r}_{1}, \boldsymbol{r}_{2}$ : Radii of the elements 1 and 2 of the stepped symmetric circular beam respectively.

$\boldsymbol{A}_{12}$ : Ratio of the across sectional areas of the elements 1 and 2 of the stepped symmetric circular beam respectively.

$\boldsymbol{r}_{12} \quad$ : Ratio of the radii of the elements 1 and 2 of the stepped symmetric circular beam respectively.

$\boldsymbol{E}_{12} \quad$ : Ratio of the modulus of elasticity of the elements 1 and 2 of the stepped symmetric circular beam respectively.

$\alpha_{12} \quad$ : Ratio of the linear coefficient of thermal expansion of the elements 1 and 2 of the stepped symmetric circular beam respectively.

$\lambda_{\boldsymbol{L M}}, \boldsymbol{\lambda}_{\boldsymbol{L T}} \quad$ : Linear mechanical $\left(\frac{P L^{2}}{E_{2} I_{2}}\right)$ and thermal critical load $\left(\frac{\alpha_{2} T L^{2}}{r_{2}{ }^{2}}\right)$ parameters respectively

$\lambda_{N L T} \quad$ : Thermal post buckling load parameter

$\lambda_{\boldsymbol{T}_{\boldsymbol{a}}} \quad:$ Axial tension parameter $\left(\frac{T_{a} L^{2}}{E_{2} I_{2}}\right)$
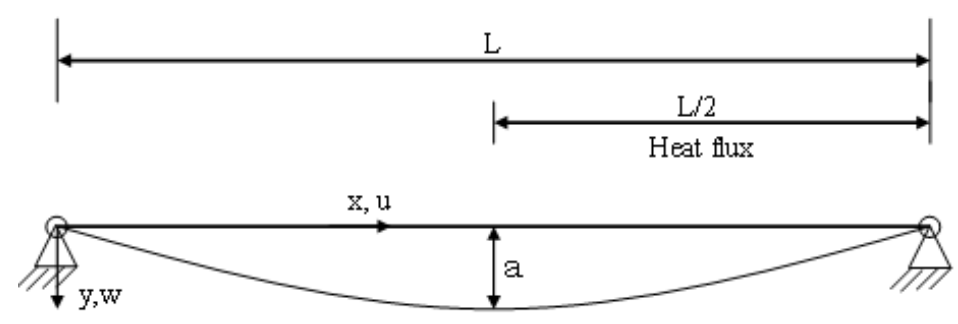

Simply supported beam

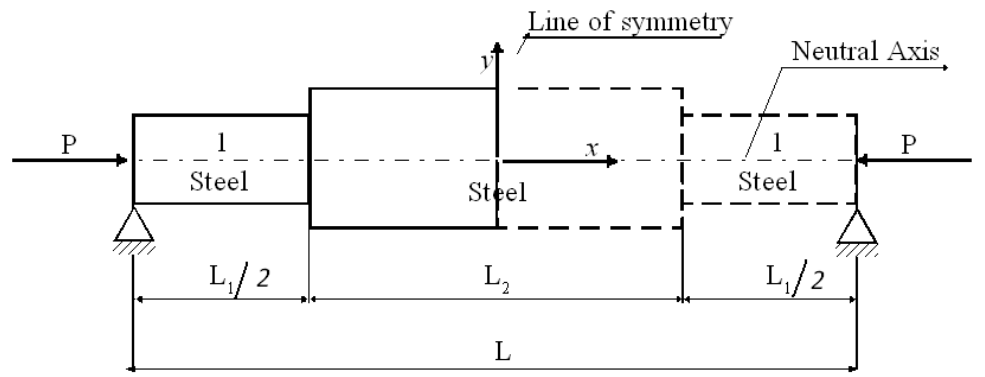

Figure 1a: Geometry of symmetric stepped steel simply supported beam

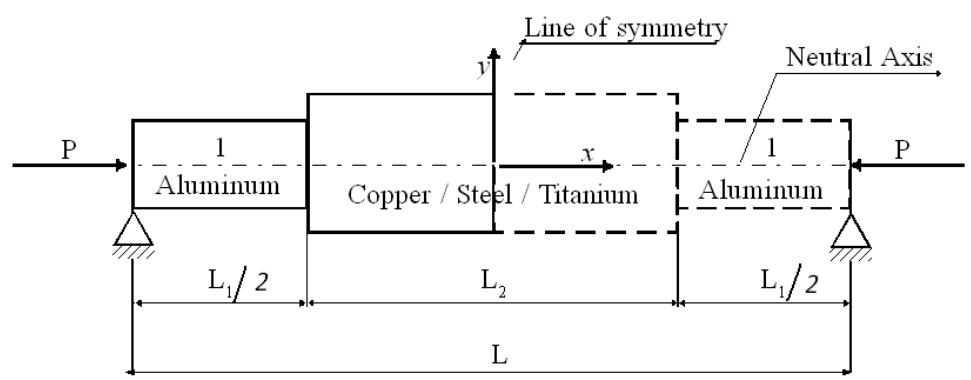

Figure 1b: Geometry of symmetric stepped compound simply supported beam with different materials 
Table 1: Thermo-Mechanical properties of aluminum, steel, copper and titanium

\begin{tabular}{|c|c|c|c|}
\hline \multirow{2}{*}{ Material } & $\begin{array}{c}\text { Young's Modulus } \\
\mathrm{GPa}\end{array}$ & $\begin{array}{c}\text { Density } \\
K g\end{array}$ & $\begin{array}{c}\text { Linear coefficient of thermal expansion } \\
\alpha\end{array}$ \\
\hline Aluminum & 69 & 2780 & $23 \times 10^{-6}$ \\
\hline Steel & 204 & 7750 & $11.76 \times 10^{-6}$ \\
\hline Copper & 110 & 8940 & $17.21 \times 10^{-6}$ \\
\hline Titanium & 115 & 4429 & $8.25 \times 10^{-6}$ \\
\hline
\end{tabular}

Table 2: indicating the Values $\left(\lambda_{N L T} / \lambda_{L T}\right)$ for uniform simply supported isotropic beam

\begin{tabular}{|c|c|c|c|c|c|}
\hline \multirow[b]{2}{*}{$a / r_{2}$} & \multicolumn{5}{|c|}{$\begin{array}{c}\frac{\lambda_{N L T}}{\lambda_{L T}} \\
\text { Uniform simply supported isotropic Beam } \\
\text { I12=1 }\end{array}$} \\
\hline & 0.1 & 0.2 & 0.3 & 0.4 & 0.5 \\
\hline 0.0 & 1.0000 & 1.0000 & 1.0000 & 1.0000 & 1.0000 \\
\hline 0.2 & $\begin{array}{c}1.0101(1.0505) \\
1.0100^{*}\end{array}$ & $\begin{array}{c}1.0101(1.0505) \\
1.0100^{*}\end{array}$ & $\begin{array}{c}1.0101(1.0505) \\
1.0100^{*}\end{array}$ & $\begin{array}{c}1.0101(1.0505) \\
1.0100^{*}\end{array}$ & $\begin{array}{c}1.0101(1.0505) \\
1.0100^{*}\end{array}$ \\
\hline 0.4 & $\begin{array}{c}1.0403(1.0819) \\
1.0400^{*}\end{array}$ & $\begin{array}{c}1.0403(1.0819) \\
1.0400^{*}\end{array}$ & $\begin{array}{c}1.0403(1.0819) \\
1.0400^{*}\end{array}$ & $\begin{array}{c}1.0403(1.0819) \\
1.0400^{*}\end{array}$ & $\begin{array}{c}1.0403(1.0819) \\
1.0400^{*}\end{array}$ \\
\hline 0.6 & $\begin{array}{c}1.0907(1.1343) \\
1.0900^{*}\end{array}$ & $\begin{array}{c}1.0907(1.1343) \\
1.0900^{*}\end{array}$ & $\begin{array}{c}1.0907(1.1343) \\
1.0900^{*}\end{array}$ & $\begin{array}{c}1.0907(1.1343) \\
1.0900^{*}\end{array}$ & $\begin{array}{c}1.0907(1.1343) \\
1.0900^{*}\end{array}$ \\
\hline 0.8 & $\begin{array}{c}1.1612(1.2076) \\
1.1600^{*}\end{array}$ & $\begin{array}{c}1.1612(1.2076) \\
1.1600^{*} \\
\end{array}$ & $\begin{array}{c}1.1612(1.2076) \\
1.1600^{*}\end{array}$ & $\begin{array}{c}1.1612(1.2076) \\
1.1600^{*}\end{array}$ & $\begin{array}{c}1.1612(1.2076) \\
1.1600^{*}\end{array}$ \\
\hline 1.0 & $\begin{array}{c}1.2519(1.3020) \\
1.2500^{*}\end{array}$ & $\begin{array}{c}1.2519(1.3020) \\
1.2500^{*}\end{array}$ & $\begin{array}{c}1.2519(1.3020) \\
1.2500^{*}\end{array}$ & $\begin{array}{c}1.2519(1.3020) \\
1.2500^{*}\end{array}$ & $\begin{array}{c}1.2519(1.3020) \\
1.2500^{*}\end{array}$ \\
\hline 2.0 & $\begin{array}{c}2.0077(2.0880) \\
2.0000^{*}\end{array}$ & $\begin{array}{c}2.0077(2.0880) \\
2.0000^{*}\end{array}$ & $\begin{array}{c}2.0077(2.0880) \\
2.0000^{*}\end{array}$ & $\begin{array}{c}2.0077(2.0880) \\
2.0000^{*}\end{array}$ & $\begin{array}{c}2.0077(2.0880) \\
2.0000^{*}\end{array}$ \\
\hline 3.0 & $\begin{array}{c}3.2674(3.3981) \\
3.2500^{*}\end{array}$ & $\begin{array}{c}3.2674(3.3981) \\
3.2500^{*}\end{array}$ & $\begin{array}{c}3.2674(3.3981) \\
3.2500^{*}\end{array}$ & $\begin{array}{c}3.2674(3.3981) \\
3.2500^{*}\end{array}$ & $\begin{array}{c}3.2674(3.3981) \\
3.2500^{*}\end{array}$ \\
\hline
\end{tabular}

* Rao. G. V. and Raju. K. K [5] values shown inside ( ) obtained by ANSYS Values shown outside ( ) obtained by theoretical analysis

Table 3: indicating the Values $\left(\lambda_{N L T} / \lambda_{L T}\right)$ for stepped simply supported isotropic beam of $I_{12}=0.1$

\begin{tabular}{|c|c|c|c|c|c|}
\hline \multirow{2}{*}{$a / r_{2}$} & \multicolumn{5}{|c|}{$\begin{array}{c}\frac{\lambda_{N L T}}{\lambda_{L T}} \\
\text { Isotropic Stepped simply supported Beam } \\
\text { I12=0.1 }\end{array}$} \\
\hline & 0.1 & 0.2 & 0.3 & 0.4 & 0.5 \\
\hline 0.0 & 1.0000 & 1.0000 & 1.0000 & 1.0000 & 1.0000 \\
\hline 0.2 & $1.0089(1.0493)$ & $1.0078(1.0481)$ & $1.0069(1.0472)$ & $1.0062(1.0464)$ & $1.0056(1.0458)$ \\
\hline 0.4 & $1.0357(1.0771)$ & $1.0313(1.0726)$ & $1.0276(1.0687)$ & $1.0247(1.0657)$ & $1.0224(1.0633)$ \\
\hline 0.6 & $1.0803(1.1235)$ & $1.0705(1.1133)$ & $1.0621(1.1046)$ & $1.0555(1.0977)$ & $1.0505(1.0925)$ \\
\hline 0.8 & $1.1428(1.1885)$ & $1.1253(1.1703)$ & $1.1105(1.1549)$ & $1.0987(1.1426)$ & $1.0897(1.1333)$ \\
\hline 1.0 & $1.2232(1.2721)$ & $1.1958(1.2436)$ & $1.1726(1.2195)$ & $1.1542(1.2004)$ & $1.1401(1.1857)$ \\
\hline 2.0 & $1.8927(1.9684)$ & $1.7833(1.8546)$ & $1.6904(1.7580)$ & $1.6166(1.6813)$ & $1.5606(1.6230)$ \\
\hline 3.0 & $3.0086(3.1289)$ & $2.7624(2.8729)$ & $2.5535(2.6556)$ & $2.3874(2.4829)$ & $2.2613(2.3518)$ \\
\hline
\end{tabular}

values shown inside () obtained by ANSYS values shown outside () obtained by theoretical analysis 
Table 4: indicating the Values $\left(\lambda_{N L T} / \lambda_{L T}\right)$ for stepped simply supported isotropic beam of $I_{12}=0.2$

\begin{tabular}{|c|c|c|c|c|c|}
\hline \multirow{2}{*}{$a / r_{2}$} & \multicolumn{5}{|c|}{$\begin{array}{c}\frac{\lambda_{N L T}}{\lambda_{L T}} \\
\text { Isotropic Stepped simply supported Beam } \\
\text { I12=0.2 }\end{array}$} \\
\hline & 0.1 & 0.2 & 0.3 & 0.4 & 0.5 \\
\hline 0.0 & 1.0000 & 1.0000 & 1.0000 & 1.0000 & 1.0000 \\
\hline 0.2 & $1.0090(1.0494)$ & $1.0080(1.0483)$ & $1.0071(1.0474)$ & $1.0063(1.0466)$ & $1.0059(1.0461)$ \\
\hline 0.4 & $1.0360(1.0774)$ & $1.0318(1.0731)$ & $1.0282(1.0693)$ & $1.0254(1.0664)$ & $1.0235(1.0664)$ \\
\hline 0.6 & $1.0810(1.1242)$ & $1.0717(1.1146)$ & $1.0635(1.1060)$ & $1.0571(1.0994)$ & $1.0529(1.0950)$ \\
\hline 0.8 & $1.1440(1.1898)$ & $1.1274(1.1725)$ & $1.1129(1.1574)$ & $1.1016(1.1457)$ & $1.0941(1.1379)$ \\
\hline 1.0 & $1.2251(1.2741)$ & $1.1990(1.2470)$ & $1.1764(1.2235)$ & $1.1587(1.2050)$ & $1.1470(1.1929)$ \\
\hline 2.0 & $1.9003(3.9763)$ & $1.7962(1.8680)$ & $1.7057(1.7739)$ & $1.6350(1.7004)$ & $1.5881(1.6516)$ \\
\hline 3.0 & $3.0256(3.1466)$ & $2.7914(2.9031)$ & $2.5879(2.6914)$ & $2.4286(2.5257)$ & $2.3233(2.4162)$ \\
\hline
\end{tabular}

values shown inside ( ) obtained by ANSYS values shown outside ( ) obtained by theoretical analysis

Table 5: indicating the Values $\left(\lambda_{N L T} / \lambda_{L T}\right)$ for stepped simply supported isotropic beam of $I_{12}=0.4$

\begin{tabular}{|c|c|c|c|c|c|}
\hline \multirow{2}{*}{$a / r_{2}$} & \multicolumn{5}{|c|}{$\begin{array}{c}\frac{\lambda_{N L T}}{\lambda_{L T}} \\
\text { Isotropic Stepped simply supported Beam } \\
\text { I12=0.4 }\end{array}$} \\
\hline & 0.1 & 0.2 & 0.3 & 0.4 & 0.5 \\
\hline 0.0 & 1.0000 & 1.0000 & 1.0000 & 1.0000 & 1.0000 \\
\hline 0.2 & $1.0092(1.0496)$ & $1.0084(1.0487)$ & $1.0077(1.0480)$ & $1.0072(1.0475)$ & $1.0069(1.0472)$ \\
\hline 0.4 & $1.0369(1.0784)$ & $1.0337(1.0750)$ & $1.0309(1.0721)$ & $1.0289(1.0701)$ & $1.0278(1.0689)$ \\
\hline 0.6 & $1.0831(1.1264)$ & $1.0758(1.1188)$ & $1.0696(1.1124)$ & $1.0650(1.1076)$ & $1.0625(1.1050)$ \\
\hline 0.8 & $1.1477(1.1936)$ & $1.1348(1.1802)$ & $1.1238(1.1688)$ & $1.1156(1.1602)$ & $1.1111(1.1555)$ \\
\hline 1.0 & $1.2308(1.2800)$ & $1.2107(1.2591)$ & $1.1934(1.2411)$ & $1.1806(1.2278)$ & $1.1736(1.2205)$ \\
\hline 2.0 & $1.9234(2.0003)$ & $1.8426(1.9163)$ & $1.7736(1.8445)$ & $1.7224(1.7913)$ & $1.6942(1.7620)$ \\
\hline 3.0 & $3.0776(3.2007)$ & $2.8959(3.0117)$ & $2.7405(2.8501)$ & $2.6255(2.7305)$ & $2.5621(2.6646)$ \\
\hline
\end{tabular}

values shown inside () obtained by ANSYS

values shown outside ( ) obtained by theoretical analysis

Table 6: indicating the Values $\left(\lambda_{N L T} / \lambda_{L T}\right)$ for stepped simply supported isotropic beam of $I_{12}=0.6$

\begin{tabular}{|c|c|c|c|c|c|}
\hline \multirow{2}{*}{$a / r_{2}$} & \multicolumn{5}{|c|}{$\begin{array}{c}\frac{\lambda_{N L T}}{\lambda_{L T}} \\
\text { Isotropic Stepped simply supported Beam } \\
\text { I12 }=0.6\end{array}$} \\
\hline & 0.1 & 0.2 & 0.3 & 0.4 & 0.5 \\
\hline 0.0 & 1.0000 & 1.0000 & 1.0000 & 1.0000 & 1.0000 \\
\hline 0.2 & $1.0095(1.0499)$ & $1.0090(1.0494)$ & $1.0085(1.0488)$ & $1.0082(1.0485)$ & $1.0080(1.0483)$ \\
\hline 0.4 & $1.0380(1.0795)$ & $1.0358(1.0772)$ & $1.0340(1.0754)$ & $1.0328(1.0741)$ & $1.0322(1.0735)$ \\
\hline 0.6 & $1.0855(1.1289)$ & $1.0807(1.1239)$ & $1.0766(1.1197)$ & $1.0737(1.1166)$ & $1.0724(1.1153)$ \\
\hline 0.8 & $1.1520(1.1981)$ & $1.1434(1.1891)$ & $1.1362(1.1816)$ & $1.1311(1.1763)$ & $1.1286(1.1737)$ \\
\hline 1.0 & $1.2375(1.2870)$ & $1.2240(1.2730)$ & $1.2128(1.2613)$ & $1.2048(1.2530)$ & $1.2010(1.2490)$ \\
\hline 2.0 & $1.9502(2.0282)$ & $1.8962(1.9720)$ & $1.8510(1.9250)$ & $1.8192(1.8920)$ & $1.8040(1.8762)$ \\
\hline 3.0 & $3.1379(3.2634)$ & $3.0163(3.1370)$ & $2.9148(3.0314)$ & $2.8431(2.9568)$ & $2.8090(2.9214)$ \\
\hline
\end{tabular}

values shown inside ( ) obtained by ANSYS values shown outside ( ) obtained by theoretical analysis

Table 7: indicating the Values $\left(\lambda_{N L T} / \lambda_{L T}\right)$ for stepped simply supported isotropic beam of $I_{12}=0.8$

\begin{tabular}{|c|c|c|c|c|c|}
\hline \multirow[t]{2}{*}{$\begin{array}{c}\zeta \\
a / r_{2}\end{array}$} & \multicolumn{5}{|c|}{$\begin{array}{c}\frac{\lambda_{N L T}}{\lambda_{L T}} \\
\text { Isotropic Stepped simply supported Beam } \\
\text { I12=0.8 }\end{array}$} \\
\hline & 0.1 & 0.2 & 0.3 & 0.4 & 0.5 \\
\hline 0.0 & 1.0000 & 1.0000 & 1.0000 & 1.0000 & 1.0000 \\
\hline 0.2 & $1.0098(1.0502)$ & $1.0095(1.0499)$ & $1.0093(1.0497)$ & $1.0091(1.0495)$ & $1.0091(1.0495)$ \\
\hline 0.4 & $1.0391(1.0807)$ & $1.0381(1.0796)$ & $1.0372(1.0787)$ & $1.0366(1.0781)$ & $1.0363(1.0778)$ \\
\hline 0.6 & $1.0881(1.1316)$ & $1.0857(1.1291)$ & $1.0837(1.1270)$ & $1.0823(1.1256)$ & $1.0818(1.1251)$ \\
\hline 0.8 & $1.1566(1.2029)$ & $1.1523(1.1984)$ & $1.1488(1.1948)$ & $1.1464(1.1923)$ & $1.1454(1.1912)$ \\
\hline 1.0 & $1.2446(1.2944)$ & $1.2379(1.2874)$ & $1.2324(1.2817)$ & $1.2287(1.2778)$ & $1.2272(1.2763)$ \\
\hline 2.0 & $1.9786(2.0577)$ & $1.9517(2.0298)$ & $1.9297(2.0069)$ & $1.9149(1.9915)$ & $1.9087(1.9850)$ \\
\hline 3.0 & $3.2017(3.3298)$ & $3.1413(3.2670)$ & $3.0919(3.2156)$ & $3.0584(3.1807)$ & $3.0445(3.1663)$ \\
\hline
\end{tabular}

values shown inside ( ) obtained by ANSYS values shown outside ( ) obtained by theoretical analysis 
Table 8: indicating the Values $\left(\lambda_{N L T} / \lambda_{L T}\right)$ for stepped simply supported compound beam of $I_{12}=0.1$

\begin{tabular}{|c|c|c|c|c|c|c|c|c|c|c|c|c|c|c|c|}
\hline & & & & & & 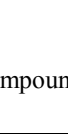 & . & $\begin{array}{l}\frac{T}{T} \\
\text { aply } \\
0.1\end{array}$ & gorted & & & & & & \\
\hline$\zeta$ & & $E_{12}=0$. & 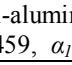 & 552 & & & $\begin{array}{r}\text { Tita } \\
E_{12}=0\end{array}$ & Im-a & $\begin{array}{l}\mathrm{m} \\
871 \\
\end{array}$ & & & $\begin{array}{r}\mathrm{C} \\
E_{12}=0 \\
\end{array}$ & & 367 & \\
\hline $\begin{array}{l}a / \\
r_{2}\end{array}$ & 0.1 & 0.2 & 0.3 & 0.4 & 0.5 & 0.1 & 0.2 & 0.3 & 0.4 & 0.5 & 0.1 & 0.2 & 0.3 & 0.4 & 0.5 \\
\hline $\begin{array}{c}0 . \\
0\end{array}$ & $\begin{array}{c}.000 \\
0\end{array}$ & & $\begin{array}{c}1.00 \\
00\end{array}$ & $\begin{array}{c}1.00 \\
00\end{array}$ & $\begin{array}{c}1.00 \\
00\end{array}$ & $\begin{array}{c}1.00 \\
00\end{array}$ & $\begin{array}{c}1.00 \\
00\end{array}$ & $\begin{array}{c}1.00 \\
00\end{array}$ & $\begin{array}{c}1.000 \\
0\end{array}$ & $\begin{array}{c}1.000 \\
0\end{array}$ & $\begin{array}{c}1.00 \\
00\end{array}$ & $\begin{array}{c}1.00 \\
00\end{array}$ & $\begin{array}{c}1.00 \\
00\end{array}$ & $\begin{array}{c}1.00 \\
00\end{array}$ & $\begin{array}{c}1.000 \\
0\end{array}$ \\
\hline $\begin{array}{l}0 . \\
2\end{array}$ & $\begin{array}{c}1.005 \\
8 \\
(1.04 \\
60)\end{array}$ & $\begin{array}{c}1.00 \\
51 \\
(1.04 \\
53)\end{array}$ & $\begin{array}{c}1.00 \\
49 \\
(1.04 \\
51)\end{array}$ & $\begin{array}{c}1.00 \\
48 \\
(1.04 \\
50)\end{array}$ & $\begin{array}{c}1.00 \\
46 \\
(1.04 \\
48)\end{array}$ & $\begin{array}{c}1.01 \\
45 \\
(1.05 \\
51) \\
\end{array}$ & $\begin{array}{c}1.01 \\
21 \\
(1.05 \\
26)\end{array}$ & $\begin{array}{c}1.01 \\
03 \\
(1.05 \\
07)\end{array}$ & $\begin{array}{c}1.009 \\
0 \\
(1.04 \\
94)\end{array}$ & $\begin{array}{c}1.008 \\
0 \\
(1.04 \\
83)\end{array}$ & $\begin{array}{c}1.00 \\
75 \\
(1.04 \\
78) \\
\end{array}$ & $\begin{array}{c}1.00 \\
65 \\
(1.04 \\
68)\end{array}$ & $\begin{array}{c}1.00 \\
58 \\
(1.04 \\
60) \\
\end{array}$ & $\begin{array}{c}1.00 \\
53 \\
(1.04 \\
55)\end{array}$ & $\begin{array}{c}1.005 \\
0 \\
(1.04 \\
52) \\
\end{array}$ \\
\hline $\begin{array}{l}0 . \\
4\end{array}$ & $\begin{array}{c}1.023 \\
2 \\
(1.06 \\
41) \\
\end{array}$ & $\begin{array}{c}1.02 \\
06 \\
(1.61 \\
4) \\
\end{array}$ & $\begin{array}{c}1.01 \\
95 \\
(1.06 \\
03) \\
\end{array}$ & $\begin{array}{c}1.01 \\
94 \\
(1.06 \\
02) \\
\end{array}$ & $\begin{array}{c}1.01 \\
91 \\
(1.05 \\
99) \\
\end{array}$ & $\begin{array}{c}1.05 \\
80 \\
(1.10 \\
03) \\
\end{array}$ & $\begin{array}{c}1.04 \\
84 \\
(1.09 \\
03) \\
\end{array}$ & $\begin{array}{c}1.04 \\
13 \\
(1.08 \\
30) \\
\end{array}$ & $\begin{array}{c}1.036 \\
1 \\
(1.07 \\
75) \\
\end{array}$ & $\begin{array}{c}1.031 \\
8 \\
(1.07 \\
31) \\
\end{array}$ & $\begin{array}{c}1.02 \\
98 \\
(1.07 \\
10) \\
\end{array}$ & $\begin{array}{c}1.02 \\
60 \\
(1.06 \\
70) \\
\end{array}$ & $\begin{array}{c}1.02 \\
33 \\
(1.06 \\
42) \\
\end{array}$ & $\begin{array}{c}1.02 \\
14 \\
(1.06 \\
23) \\
\end{array}$ & $\begin{array}{c}1.020 \\
0 \\
(1.06 \\
08) \\
\end{array}$ \\
\hline $\begin{array}{l}0 . \\
6\end{array}$ & $\begin{array}{c}1.052 \\
3 \\
(1.09 \\
44) \\
\end{array}$ & $\begin{array}{c}1.04 \\
63 \\
(1.08 \\
82) \\
\end{array}$ & $\begin{array}{c}1.04 \\
39 \\
(1.08 \\
57) \\
\end{array}$ & $\begin{array}{c}1.04 \\
36 \\
(1.08 \\
53) \\
\end{array}$ & $\begin{array}{c}1.04 \\
29 \\
(1.08 \\
46) \\
\end{array}$ & $\begin{array}{c}1.13 \\
04 \\
(1.17 \\
6) \\
\end{array}$ & $\begin{array}{c}1.10 \\
89 \\
(1.15 \\
33) \\
\end{array}$ & $\begin{array}{c}1.09 \\
30 \\
(1.13 \\
67) \\
\end{array}$ & $\begin{array}{c}1.081 \\
3 \\
(1.12 \\
46) \\
\end{array}$ & $\begin{array}{c}1.071 \\
6 \\
(1.11 \\
45) \\
\end{array}$ & $\begin{array}{c}1.06 \\
72 \\
(1.10 \\
99) \\
\end{array}$ & $\begin{array}{c}1.05 \\
86 \\
(1.10 \\
09) \\
\end{array}$ & $\begin{array}{c}1.05 \\
24 \\
(1.09 \\
45) \\
\end{array}$ & $\begin{array}{c}1.04 \\
81 \\
(1.09 \\
00) \\
\end{array}$ & $\begin{array}{c}1.044 \\
9 \\
(1.08 \\
67) \\
\end{array}$ \\
\hline $\begin{array}{l}0 . \\
8\end{array}$ & $\begin{array}{c}1.092 \\
9 \\
(1.13 \\
66)\end{array}$ & $\begin{array}{c}1.08 \\
24 \\
(1.12 \\
57)\end{array}$ & $\begin{array}{c}1.07 \\
81 \\
(1.12 \\
12)\end{array}$ & $\begin{array}{c}1.07 \\
74 \\
(1.12 \\
05) \\
\end{array}$ & $\begin{array}{c}1.07 \\
63 \\
(1.11 \\
94)\end{array}$ & $\begin{array}{c}1.23 \\
18 \\
(1.28 \\
11) \\
\end{array}$ & $\begin{array}{c}1.19 \\
36 \\
(1.24 \\
13)\end{array}$ & $\begin{array}{c}1.16 \\
54 \\
(1.21 \\
20) \\
\end{array}$ & $\begin{array}{c}1.144 \\
5 \\
(1.19 \\
03)\end{array}$ & $\begin{array}{c}1.127 \\
3 \\
(1.17 \\
24)\end{array}$ & $\begin{array}{c}1.11 \\
94 \\
(1.16 \\
42) \\
\end{array}$ & $\begin{array}{c}1.10 \\
41 \\
(1.14 \\
83)\end{array}$ & $\begin{array}{c}1.09 \\
31 \\
(1.13 \\
68) \\
\end{array}$ & $\begin{array}{c}1.08 \\
56 \\
(1.12 \\
90) \\
\end{array}$ & $\begin{array}{c}1.079 \\
8 \\
(1.12 \\
30) \\
\end{array}$ \\
\hline $\begin{array}{l}1 . \\
0\end{array}$ & $\begin{array}{c}1.145 \\
2 \\
(1.19 \\
10)\end{array}$ & $\begin{array}{c}1.12 \\
87 \\
(1.17 \\
38)\end{array}$ & $\begin{array}{c}1.12 \\
20 \\
(1.16 \\
69)\end{array}$ & $\begin{array}{c}1.12 \\
10 \\
(1.16 \\
58)\end{array}$ & $\begin{array}{c}1.11 \\
92 \\
(1.16 \\
40)\end{array}$ & $\begin{array}{c}1.36 \\
23 \\
(1.41 \\
68)\end{array}$ & $\begin{array}{c}1.30 \\
26 \\
(1.35 \\
47)\end{array}$ & $\begin{array}{c}1.25 \\
84 \\
(1.30 \\
87)\end{array}$ & $\begin{array}{c}1.225 \\
9 \\
(1.27 \\
49)\end{array}$ & $\begin{array}{c}1.198 \\
9 \\
(1.24 \\
69)\end{array}$ & $\begin{array}{c}1.18 \\
65 \\
(1.23 \\
40)\end{array}$ & $\begin{array}{c}1.16 \\
27 \\
(1.20 \\
92)\end{array}$ & $\begin{array}{c}1.14 \\
55 \\
(1.19 \\
13)\end{array}$ & $\begin{array}{c}1.13 \\
37 \\
(1.17 \\
90)\end{array}$ & $\begin{array}{c}1.124 \\
7 \\
(1.16 \\
97)\end{array}$ \\
\hline $\begin{array}{l}2 . \\
0\end{array}$ & $\begin{array}{c}1.580 \\
8 \\
(1.64 \\
40) \\
\end{array}$ & $\begin{array}{c}1.51 \\
48 \\
(1.57 \\
54) \\
\end{array}$ & $\begin{array}{c}1.48 \\
81 \\
(1.54 \\
76) \\
\end{array}$ & $\begin{array}{c}1.48 \\
39 \\
(1.54 \\
33) \\
\end{array}$ & $\begin{array}{c}1.47 \\
67 \\
(1.53 \\
58) \\
\end{array}$ & $\begin{array}{c}2.44 \\
90 \\
(2.54 \\
70) \\
\end{array}$ & $\begin{array}{c}2.21 \\
03 \\
(2.29 \\
87) \\
\end{array}$ & $\begin{array}{c}2.03 \\
36 \\
(2.11 \\
49) \\
\end{array}$ & $\begin{array}{c}1.903 \\
4 \\
(1.97 \\
95) \\
\end{array}$ & $\begin{array}{c}1.795 \\
5 \\
(1.86 \\
73) \\
\end{array}$ & $\begin{array}{c}1.74 \\
62 \\
(1.81 \\
60) \\
\end{array}$ & $\begin{array}{c}1.65 \\
07 \\
(1.71 \\
67) \\
\end{array}$ & $\begin{array}{c}1.58 \\
19 \\
(1.64 \\
52) \\
\end{array}$ & $\begin{array}{c}1.53 \\
50 \\
(1.59 \\
64) \\
\end{array}$ & $\begin{array}{c}1.499 \\
0 \\
(1.55 \\
90) \\
\end{array}$ \\
\hline $\begin{array}{l}3 . \\
0\end{array}$ & $\begin{array}{c}2.306 \\
7 \\
(2.39 \\
90)\end{array}$ & $\begin{array}{c}2.15 \\
83 \\
(2.24 \\
46)\end{array}$ & $\begin{array}{c}2.09 \\
82 \\
(2.18 \\
21)\end{array}$ & $\begin{array}{c}2.08 \\
88 \\
(2.17 \\
24)\end{array}$ & $\begin{array}{c}2.07 \\
25 \\
(2.15 \\
54)\end{array}$ & $\begin{array}{c}4.26 \\
04 \\
(4.43 \\
08)\end{array}$ & $\begin{array}{c}3.72 \\
32 \\
(3.87 \\
21)\end{array}$ & $\begin{array}{c}3.32 \\
55 \\
(3.45 \\
85)\end{array}$ & $\begin{array}{c}3.032 \\
7 \\
(3.15 \\
40)\end{array}$ & $\begin{array}{c}2.789 \\
9 \\
(2.90 \\
15)\end{array}$ & $\begin{array}{c}2.67 \\
89 \\
(2.78 \\
61)\end{array}$ & $\begin{array}{c}2.46 \\
41 \\
(2.56 \\
27)\end{array}$ & $\begin{array}{c}2.30 \\
92 \\
(2.40 \\
16)\end{array}$ & $\begin{array}{c}2.20 \\
36 \\
(2.29 \\
17)\end{array}$ & $\begin{array}{c}2.122 \\
7 \\
(2.20 \\
76)\end{array}$ \\
\hline
\end{tabular}

values shown inside ( ) obtained by ANSYS values shown outside ( ) obtained by theoretical analysis

Table 9: indicating the Values $\left(\lambda_{N L T} / \lambda_{L T}\right)$ for stepped simply supported compound beam of

$$
I_{12}=0.2
$$

\begin{tabular}{|c|c|c|c|c|c|c|c|c|c|c|c|c|c|c|c|}
\hline \multicolumn{16}{|c|}{$\begin{array}{c}\frac{\lambda_{N L T}}{\lambda_{L T}} \\
\text { Compound stepped simply supported Beam } \\
I_{12=0.2} \\
\end{array}$} \\
\hline \multirow[b]{2}{*}{$a / r_{2}$} & \multicolumn{5}{|c|}{$\begin{array}{c}\text { Steel-aluminum } \\
E_{12}=0.34459, \alpha_{12}=1.9552\end{array}$} & \multicolumn{5}{|c|}{$\begin{array}{c}\text { Titanium-aluminum } \\
E_{12}=0.6182, \alpha_{12}=2.7871\end{array}$} & \multicolumn{5}{|c|}{$\begin{array}{c}\text { Copper-aluminum } \\
E_{12}=0.6375, \alpha_{12}=1.3367\end{array}$} \\
\hline & 0.1 & 0.2 & 0.3 & 0.4 & 0.5 & 0.1 & 0.2 & 0.3 & 0.4 & 0.5 & 0.1 & 0.2 & 0.3 & 0.4 & 0.5 \\
\hline \multirow{2}{*}{0.0} & 1.00 & 1.000 & 1.000 & 1.000 & 1.000 & 1.000 & 1.000 & 1.000 & 1.000 & 1.000 & 1.000 & 1.000 & 1.000 & 1.000 & 1.000 \\
\hline & 00 & 0 & 0 & 0 & 0 & 0 & 0 & 0 & 0 & 0 & 0 & 0 & 0 & 0 & 0 \\
\hline \multirow{4}{*}{0.2} & 1.00 & 1.005 & 1.004 & 1.004 & 1.004 & 1.014 & 1.012 & 1.010 & 1.008 & 1.007 & 1.007 & 1.006 & 1.005 & 1.005 & 1.004 \\
\hline & 58 & 0 & 6 & 3 & 2 & 5 & 1 & 2 & 7 & 7 & 5 & 5 & 7 & 2 & 8 \\
\hline & (1.04 & (1.045 & (1.04 & (1.04 & (1.04 & (1.05 & (1.05 & (1.05 & (1.04 & (1.04 & (1.04 & (1.04 & (1.04 & (1.04 & (1.045 \\
\hline & 60) & 2) & 48) & 45) & 44) & 51) & 26) & 06) & 90) & 80) & 78) & 68) & 59) & 54) & 0) \\
\hline \multirow{4}{*}{0.4} & 1.02 & 1.020 & 1.018 & 1.017 & 1.016 & 1.058 & 1.048 & 1.040 & 1.034 & 1.030 & 1.030 & 1.026 & 1.023 & 1.020 & 1.019 \\
\hline & 32 & 2 & 3 & 4 & 7 & 2 & 4 & 7 & 9 & 7 & 0 & 1 & 0 & 8 & 4 \\
\hline & (1.06 & (1.061 & (1.05 & (1.05 & (1.05 & $(1.10$ & (1.09 & (1.08 & (1.07 & (1.07 & (1.07 & (1.06 & (1.06 & (1.06 & (1.060 \\
\hline & 41) & 0) & 90) & 81) & 74) & 05) & 03) & 23) & 63) & 19) & 12) & 71) & 39) & 16) & 2) \\
\hline \multirow{4}{*}{0.6} & 1.05 & 1.045 & 1.041 & 1.039 & 1.037 & 1.130 & 1.108 & 1.091 & 1.078 & 1.069 & 1.067 & 1.058 & 1.051 & 1.046 & 1.043 \\
\hline & 23 & 4 & 3 & 1 & 7 & 9 & 9 & 6 & 6 & 1 & 4 & 6 & 7 & 7 & 6 \\
\hline & (1.09 & (1.087 & (1.08 & (1.08 & (1.07 & (1.17 & $(1.15$ & (1.13 & (1.12 & (1.11 & (1.11 & $(1.10$ & (1.09 & (1.08 & (1.085 \\
\hline & 44) & 2) & 30) & 07) & 92) & 61) & 33) & 53) & 17) & 19) & 01) & 09) & 38) & 86) & 3) \\
\hline \multirow{2}{*}{0.8} & 1.09 & 1.080 & 1.073 & 1.069 & 1.067 & 1.232 & 1.193 & 1.162 & 1.139 & 1.122 & 1.119 & 1.104 & 1.091 & 1.083 & 1.077 \\
\hline & 29 & 7 & 3 & 5 & 0 & 8 & 7 & 8 & 7 & 8 & 9 & 2 & 9 & 1 & 4 \\
\hline
\end{tabular}




\begin{tabular}{|c|c|c|c|c|c|c|c|c|c|c|c|c|c|c|c|}
\hline & $\begin{array}{c}(1.13 \\
66) \\
\end{array}$ & $\begin{array}{c}(1.123 \\
9)\end{array}$ & $\begin{array}{c}(1.11 \\
62) \\
\end{array}$ & $\begin{array}{c}(1.11 \\
23)\end{array}$ & $\begin{array}{c}(1.10 \\
97)\end{array}$ & $\begin{array}{c}(1.28 \\
21)\end{array}$ & $\begin{array}{c}(1.24 \\
14)\end{array}$ & $\begin{array}{c}(1.20 \\
93)\end{array}$ & $\begin{array}{c}(1.18 \\
53)\end{array}$ & $\begin{array}{c}(1.16 \\
77)\end{array}$ & $\begin{array}{c}(1.16 \\
47)\end{array}$ & $\begin{array}{c}(1.14 \\
84)\end{array}$ & $\begin{array}{c}(1.13 \\
56)\end{array}$ & $\begin{array}{c}(1.12 \\
64)\end{array}$ & $\begin{array}{c}(1.120 \\
5)\end{array}$ \\
\hline \multirow{4}{*}{1.0} & 1.14 & 1.126 & 1.114 & 1.108 & 1.104 & 1.363 & 1.302 & 1.254 & 1.218 & 1.191 & 1.187 & 1.162 & 1.143 & 1.129 & 1.121 \\
\hline & 51 & 2 & 6 & 5 & 6 & 7 & 6 & 4 & 3 & 9 & 3 & 9 & 6 & 8 & 0 \\
\hline & (1.19 & (1.171 & (1.15 & (1.15 & (1.14 & (1.41 & $(1.35$ & (1.30 & (1.26 & (1.23 & $(1.23$ & $(1.20$ & (1.18 & (1.17 & (1.165 \\
\hline & 09) & 2) & 92) & 28) & 88) & 82) & 47) & 46) & 70) & 96) & 48) & 94) & 93) & $50)$ & 8) \\
\hline \multirow{4}{*}{2.0} & 1.58 & 1.504 & 1.458 & 1.434 & 1.418 & 2.454 & 2.210 & 2.017 & 1.873 & 1.767 & 1.749 & 1.651 & 1.574 & 1.519 & 1.484 \\
\hline & 06 & 7 & 4 & 1 & 4 & 8 & 4 & 7 & 3 & 5 & 3 & 4 & 3 & 2 & 0 \\
\hline & (1.64 & (1.564 & (1.51 & (1.49 & $(1.47$ & $(2.55$ & $(2.29$ & (2.09 & (1.94 & $(1.83$ & $(1.81$ & $(1.71$ & (1.63 & (1.58 & (1.543 \\
\hline & 38) & 9) & 67) & 15) & 51) & 30) & 88) & 84) & 82) & 82) & 93) & 75) & 73) & 00) & 4) \\
\hline \multirow{4}{*}{3.0} & 2.30 & 2.135 & 2.031 & 1.976 & 1.941 & 4.273 & 3.723 & 3.289 & 2.964 & 2.726 & 2.686 & 2.465 & 2.292 & 2.168 & 2.088 \\
\hline & 63 & 5 & 5 & 7 & 5 & 3 & 4 & 8 & 9 & 8 & 0 & 7 & 2 & 2 & 9 \\
\hline & (2.39 & $(2.220$ & $(2.11$ & $(2.05$ & $(2.01$ & $(4.44$ & (3.87 & (3.42 & (3.08 & $(2.83$ & $(2.79$ & $(2.56$ & $(2.38$ & $(2.25$ & (2.172 \\
\hline & 86) & 9) & 28) & 58) & 92) & 42) & 23) & 14) & 35) & 59) & 34) & 43) & 39) & 49) & 5) \\
\hline
\end{tabular}

values shown inside ( ) obtained by ANSYS values shown outside ( ) obtained by theoretical analysis

Table 10: indicating the Values $\left(\lambda_{N L T} / \lambda_{L T}\right)$ for stepped simply supported compound beam of $I_{12}=0.4$

\begin{tabular}{|c|c|c|c|c|c|c|c|c|c|c|c|c|c|c|c|}
\hline & & & & & & ompou & steppe & $\begin{array}{l}\lambda_{N L T} \\
\lambda_{L T} \\
\text { simply } \\
12=0.4\end{array}$ & ported & & & & & & \\
\hline$\zeta$ & & $E_{12}=0$. & $\begin{array}{l}\text { alumi } \\
59, \alpha_{1}\end{array}$ & 9552 & & & $\begin{array}{r}\text { Titar } \\
E_{12}=0 .\end{array}$ & $\begin{array}{l}\text { um-alu } \\
182, \alpha\end{array}$ & $\begin{array}{l}\text { um } \\
7871 \\
\end{array}$ & & & $\begin{array}{r}\mathrm{Cc} \\
E_{12}=0 \\
\end{array}$ & $\begin{array}{l}\text { er-alu } \\
75, \alpha\end{array}$ & $\begin{array}{l}\mathrm{m} \\
3367 \\
\end{array}$ & \\
\hline $\begin{array}{l}\text { a/ } \\
r_{2}\end{array}$ & 0.1 & 0.2 & 0.3 & 0.4 & 0.5 & 0.1 & 0.2 & 0.3 & 0.4 & 0.5 & 0.1 & 0.2 & 0.3 & 0.4 & 0.5 \\
\hline $\begin{array}{l}0 . \\
0\end{array}$ & $\begin{array}{c}1.000 \\
0\end{array}$ & $\begin{array}{c}1.000 \\
0\end{array}$ & $\begin{array}{c}1.000 \\
0\end{array}$ & $\begin{array}{c}1.000 \\
0\end{array}$ & $\begin{array}{c}1.000 \\
0\end{array}$ & $\begin{array}{c}1.000 \\
0\end{array}$ & $\begin{array}{c}1.000 \\
0\end{array}$ & $\begin{array}{c}1.000 \\
0\end{array}$ & $\begin{array}{c}1.000 \\
0\end{array}$ & $\begin{array}{c}1.000 \\
0\end{array}$ & $\begin{array}{c}1.000 \\
0\end{array}$ & $\begin{array}{c}1.000 \\
0\end{array}$ & $\begin{array}{c}1.000 \\
0\end{array}$ & $\begin{array}{c}1.000 \\
0\end{array}$ & 1.0000 \\
\hline $\begin{array}{l}0 . \\
2\end{array}$ & $\begin{array}{c}1.005 \\
8 \\
(1.04 \\
60)\end{array}$ & $\begin{array}{c}1.005 \\
1 \\
(1.04 \\
53)\end{array}$ & $\begin{array}{c}1.004 \\
5 \\
(1.04 \\
47)\end{array}$ & $\begin{array}{c}1.004 \\
2 \\
(1.04 \\
44)\end{array}$ & $\begin{array}{c}1.004 \\
1 \\
(1.04 \\
43)\end{array}$ & $\begin{array}{c}1.014 \\
8 \\
(1.05 \\
54)\end{array}$ & $\begin{array}{c}1.012 \\
5 \\
(1.05 \\
30)\end{array}$ & $\begin{array}{c}1.010 \\
6 \\
(1.05 \\
10)\end{array}$ & $\begin{array}{c}1.009 \\
2 \\
(1.04 \\
96)\end{array}$ & $\begin{array}{c}1.008 \\
3 \\
(1.04 \\
86)\end{array}$ & $\begin{array}{c}1.007 \\
6 \\
(1.04 \\
79)\end{array}$ & $\begin{array}{c}1.006 \\
7 \\
(1.04 \\
70)\end{array}$ & $\begin{array}{c}1.006 \\
0 \\
(1.04 \\
62)\end{array}$ & $\begin{array}{c}1.005 \\
5 \\
(1.04 \\
57)\end{array}$ & $\begin{array}{c}1.0053 \\
(1.0455 \\
\quad)\end{array}$ \\
\hline $\begin{array}{l}0 . \\
4\end{array}$ & $\begin{array}{c}1.023 \\
4 \\
(1.06 \\
43)\end{array}$ & $\begin{array}{c}1.020 \\
3 \\
(1.06 \\
11)\end{array}$ & $\begin{array}{c}1.018 \\
1 \\
(1.05 \\
88)\end{array}$ & $\begin{array}{c}1.016 \\
8 \\
(1.05 \\
75)\end{array}$ & $\begin{array}{c}1.016 \\
3 \\
(1.05 \\
70)\end{array}$ & $\begin{array}{c}1.059 \\
1 \\
(1.10 \\
15)\end{array}$ & $\begin{array}{c}1.049 \\
9 \\
(1.09 \\
19)\end{array}$ & $\begin{array}{c}1.042 \\
5 \\
(1.08 \\
42)\end{array}$ & $\begin{array}{c}1.037 \\
0 \\
(1.07 \\
85)\end{array}$ & $\begin{array}{c}1.033 \\
3 \\
(1.07 \\
46)\end{array}$ & $\begin{array}{c}1.030 \\
4 \\
(1.07 \\
16)\end{array}$ & $\begin{array}{c}1.026 \\
9 \\
(1.06 \\
80)\end{array}$ & $\begin{array}{c}1.024 \\
1 \\
(1.06 \\
51)\end{array}$ & $\begin{array}{c}1.022 \\
1 \\
(1.06 \\
30)\end{array}$ & $\begin{array}{c}1.0211 \\
(1.0619 \\
)\end{array}$ \\
\hline $\begin{array}{l}0 . \\
6\end{array}$ & $\begin{array}{c}1.052 \\
6 \\
(1.09 \\
47)\end{array}$ & $\begin{array}{c}1.045 \\
6 \\
(1.08 \\
74)\end{array}$ & $\begin{array}{c}1.040 \\
7 \\
(1.08 \\
23)\end{array}$ & $\begin{array}{c}1.037 \\
8 \\
(1.07 \\
93)\end{array}$ & $\begin{array}{c}1.036 \\
6 \\
(1.07 \\
81)\end{array}$ & $\begin{array}{c}1.132 \\
9 \\
(1.17 \\
82)\end{array}$ & $\begin{array}{c}1.112 \\
3 \\
(1.15 \\
68)\end{array}$ & $\begin{array}{c}1.095 \\
6 \\
(1.13 \\
94)\end{array}$ & $\begin{array}{c}1.083 \\
2 \\
(1.12 \\
65)\end{array}$ & $\begin{array}{c}1.074 \\
9 \\
(1.11 \\
79)\end{array}$ & $\begin{array}{c}1.068 \\
5 \\
(1.11 \\
12)\end{array}$ & $\begin{array}{c}1.060 \\
5 \\
(1.10 \\
29)\end{array}$ & $\begin{array}{c}1.054 \\
1 \\
(1.09 \\
63)\end{array}$ & $\begin{array}{c}1.049 \\
7 \\
(1.09 \\
17)\end{array}$ & $\begin{array}{c}1.0475 \\
(1.0894 \\
\quad)\end{array}$ \\
\hline $\begin{array}{l}0 . \\
8\end{array}$ & $\begin{array}{c}1.093 \\
5 \\
(1.13 \\
72)\end{array}$ & $\begin{array}{c}1.081 \\
0 \\
(1.12 \\
42)\end{array}$ & $\begin{array}{c}1.072 \\
3 \\
(1.11 \\
52)\end{array}$ & $\begin{array}{c}1.067 \\
2 \\
(1.10 \\
99)\end{array}$ & $\begin{array}{c}1.065 \\
0 \\
(1.10 \\
76)\end{array}$ & $\begin{array}{c}1.236 \\
3 \\
(1.28 \\
58)\end{array}$ & $\begin{array}{c}1.199 \\
6 \\
(1.24 \\
76)\end{array}$ & $\begin{array}{c}1.170 \\
0 \\
(1.21 \\
68)\end{array}$ & $\begin{array}{c}1.147 \\
9 \\
(1.19 \\
38)\end{array}$ & $\begin{array}{c}1.133 \\
2 \\
(1.17 \\
85)\end{array}$ & $\begin{array}{c}1.121 \\
8 \\
(1.16 \\
67)\end{array}$ & $\begin{array}{c}1.107 \\
6 \\
(1.15 \\
19)\end{array}$ & $\begin{array}{c}1.096 \\
2 \\
(1.14 \\
00)\end{array}$ & $\begin{array}{c}1.088 \\
4 \\
(1.13 \\
19)\end{array}$ & $\begin{array}{c}1.0845 \\
(1.1279 \\
)\end{array}$ \\
\hline $\begin{array}{l}1 . \\
0\end{array}$ & $\begin{array}{c}1.146 \\
1 \\
(1.19 \\
19)\end{array}$ & $\begin{array}{c}1.126 \\
6 \\
(1.17 \\
17)\end{array}$ & $\begin{array}{c}1.113 \\
0 \\
(1.15 \\
75)\end{array}$ & $\begin{array}{c}1.105 \\
1 \\
(1.14 \\
93)\end{array}$ & $\begin{array}{c}1.101 \\
6 \\
(1.14 \\
57)\end{array}$ & $\begin{array}{c}1.369 \\
3 \\
(1.42 \\
41)\end{array}$ & $\begin{array}{c}1.311 \\
9 \\
(1.36 \\
44)\end{array}$ & $\begin{array}{c}1.265 \\
6 \\
(1.31 \\
62)\end{array}$ & $\begin{array}{c}1.231 \\
1 \\
(1.28 \\
03)\end{array}$ & $\begin{array}{c}1.208 \\
1 \\
(1.25 \\
64)\end{array}$ & $\begin{array}{c}1.190 \\
3 \\
(1.23 \\
79)\end{array}$ & $\begin{array}{c}1.168 \\
1 \\
(1.21 \\
48)\end{array}$ & $\begin{array}{c}1.150 \\
3 \\
(1.19 \\
63)\end{array}$ & $\begin{array}{c}1.138 \\
1 \\
(1.18 \\
36)\end{array}$ & $\begin{array}{c}1.1320 \\
(1.1773 \\
)\end{array}$ \\
\hline $\begin{array}{l}2 . \\
0\end{array}$ & $\begin{array}{c}1.584 \\
6 \\
(1.64 \\
80)\end{array}$ & $\begin{array}{c}1.506 \\
4 \\
(1.56 \\
67)\end{array}$ & $\begin{array}{c}1.452 \\
1 \\
(1.51 \\
02)\end{array}$ & $\begin{array}{c}1.420 \\
3 \\
(1.47 \\
71)\end{array}$ & $\begin{array}{c}1.406 \\
4 \\
(1.46 \\
27)\end{array}$ & $\begin{array}{c}2.477 \\
2 \\
(2.57 \\
63)\end{array}$ & $\begin{array}{c}2.247 \\
7 \\
(2.33 \\
76)\end{array}$ & $\begin{array}{c}2.062 \\
4 \\
(2.11 \\
49)\end{array}$ & $\begin{array}{c}1.924 \\
5 \\
(2.00 \\
15)\end{array}$ & $\begin{array}{c}1.832 \\
5 \\
(1.90 \\
58)\end{array}$ & $\begin{array}{c}1.761 \\
2 \\
(1.83 \\
16)\end{array}$ & $\begin{array}{c}1.672 \\
5 \\
(1.73 \\
94)\end{array}$ & $\begin{array}{c}1.601 \\
4 \\
(1.66 \\
55)\end{array}$ & $\begin{array}{c}1.552 \\
3 \\
(1.61 \\
44)\end{array}$ & $\begin{array}{c}1.5280 \\
(1.5891 \\
)\end{array}$ \\
\hline $\begin{array}{l}3 . \\
0\end{array}$ & $\begin{array}{c}2.315 \\
3 \\
(2.40 \\
79)\end{array}$ & $\begin{array}{c}2.139 \\
5 \\
(2.22 \\
51)\end{array}$ & $\begin{array}{c}2.017 \\
3 \\
(2.09 \\
8)\end{array}$ & $\begin{array}{c}1.945 \\
6 \\
(2.02 \\
34)\end{array}$ & $\begin{array}{c}1.914 \\
3 \\
(1.99 \\
09)\end{array}$ & $\begin{array}{c}4.323 \\
6 \\
(4.49 \\
65)\end{array}$ & $\begin{array}{c}3.807 \\
4 \\
(3.95 \\
97)\end{array}$ & $\begin{array}{c}3.390 \\
4 \\
(3.52 \\
60)\end{array}$ & $\begin{array}{c}3.080 \\
2 \\
(3.20 \\
34)\end{array}$ & $\begin{array}{c}2.873 \\
1 \\
(2.98 \\
80)\end{array}$ & $\begin{array}{c}2.712 \\
8 \\
(2.82 \\
13)\end{array}$ & $\begin{array}{c}2.513 \\
2 \\
(2.61 \\
37)\end{array}$ & $\begin{array}{c}2.353 \\
1 \\
(2.44 \\
72)\end{array}$ & $\begin{array}{c}2.242 \\
6 \\
(2.33 \\
23)\end{array}$ & $\begin{array}{c}2.1879 \\
(2.2754 \\
)\end{array}$ \\
\hline
\end{tabular}

values shown inside ( ) obtained by ANSYS values shown outside ( ) obtained by theoretical analysis 
Table 11: indicating the Values $\left(\lambda_{N L T} / \lambda_{L T}\right)$ for stepped simply supported compound beam of $I_{12}=0.6$

\begin{tabular}{|c|c|c|c|c|c|c|c|c|c|c|c|c|c|c|c|}
\hline & & & & & & npou & steppec & $\begin{array}{l}\frac{V L T}{L T} \\
\text { imply sL } \\
=0.6\end{array}$ & ported & & & & & & \\
\hline$\zeta$ & & $E_{12}=0.3$ & $\begin{array}{l}\text {-alumi } \\
59, \alpha_{1}\end{array}$ & $\begin{array}{l}n \\
.9552\end{array}$ & & & $\begin{array}{r}\text { Titar } \\
E_{12}=0 .\end{array}$ & $\begin{array}{l}\text { Im-alum } \\
82, \alpha_{12}\end{array}$ & $\begin{array}{l}\text { num } \\
.7871 \\
\end{array}$ & & & $\begin{array}{r}\text { Cop } \\
E_{12}=0 . \\
\end{array}$ & $\begin{array}{l}\text { er-alum } \\
75, \alpha_{12}\end{array}$ & Im 3367 & \\
\hline $\begin{array}{l}a / \\
r_{2}\end{array}$ & 0.1 & 0.2 & 0.3 & 0.4 & 0.5 & 0.1 & 0.2 & 0.3 & 0.4 & 0.5 & 0.1 & 0.2 & 0.3 & 0.4 & 0.5 \\
\hline 0. & 1.00 & 1.000 & 1.000 & 1.000 & 1.000 & 1.000 & 1.000 & 1.000 & 1.000 & 1.000 & 1.000 & 1.000 & 1.000 & 1.000 & 1.000 \\
\hline 0 & 00 & 0 & 0 & 0 & 0 & 0 & 0 & 0 & 0 & 0 & 0 & 0 & 0 & 0 & 0 \\
\hline $\begin{array}{l}0 . \\
2\end{array}$ & $\begin{array}{c}1.00 \\
59 \\
(1.04 \\
61)\end{array}$ & $\begin{array}{c}1.005 \\
2 \\
(1.04 \\
54)\end{array}$ & $\begin{array}{c}1.004 \\
6 \\
(1.04 \\
48)\end{array}$ & $\begin{array}{c}1.004 \\
3 \\
(1.04 \\
45)\end{array}$ & $\begin{array}{c}1.004 \\
2 \\
(1.04 \\
44)\end{array}$ & $\begin{array}{c}1.015 \\
0 \\
(1.05 \\
56)\end{array}$ & $\begin{array}{c}1.013 \\
(1.05 \\
35)\end{array}$ & $\begin{array}{c}1.011 \\
3 \\
(1.05 \\
18)\end{array}$ & $\begin{array}{c}1.010 \\
1 \\
(1.05 \\
05)\end{array}$ & $\begin{array}{c}1.009 \\
2 \\
(1.04 \\
96)\end{array}$ & $\begin{array}{c}1.007 \\
8 \\
(1.04 \\
81)\end{array}$ & $\begin{array}{c}1.007 \\
(1.04 \\
73)\end{array}$ & $\begin{array}{c}1.006 \\
4 \\
(1.04 \\
67)\end{array}$ & $\begin{array}{c}1.006 \\
0 \\
(1.04 \\
62)\end{array}$ & $\begin{array}{c}1.005 \\
9 \\
(1.04 \\
61)\end{array}$ \\
\hline $\begin{array}{l}0 . \\
4\end{array}$ & $\begin{array}{c}1.02 \\
36 \\
(1.06 \\
45) \\
\end{array}$ & $\begin{array}{c}1.020 \\
6 \\
(1.06 \\
14) \\
\end{array}$ & $\begin{array}{c}1.018 \\
5 \\
(1.05 \\
92) \\
\end{array}$ & $\begin{array}{c}1.017 \\
3 \\
(1.05 \\
80) \\
\end{array}$ & $\begin{array}{c}1.016 \\
9 \\
(1.05 \\
76) \\
\end{array}$ & $\begin{array}{c}1.060 \\
2 \\
(1.10 \\
26) \\
\end{array}$ & $\begin{array}{c}1.051 \\
9 \\
(1.09 \\
40) \\
\end{array}$ & $\begin{array}{c}1.045 \\
2 \\
(1.08 \\
70) \\
\end{array}$ & $\begin{array}{c}1.040 \\
2 \\
(1.08 \\
18) \\
\end{array}$ & $\begin{array}{c}1.036 \\
9 \\
(1.07 \\
84) \\
\end{array}$ & $\begin{array}{c}1.031 \\
0 \\
(1.07 \\
22) \\
\end{array}$ & $\begin{array}{c}1.028 \\
(1.06 \\
91)\end{array}$ & $\begin{array}{c}1.025 \\
6 \\
(1.06 \\
66) \\
\end{array}$ & $\begin{array}{c}1.024 \\
1 \\
(1.06 \\
51) \\
\end{array}$ & $\begin{array}{c}1.023 \\
5 \\
(1.06 \\
44) \\
\end{array}$ \\
\hline $\begin{array}{l}0 . \\
6\end{array}$ & $\begin{array}{c}1.05 \\
31 \\
(1.09 \\
52)\end{array}$ & $\begin{array}{c}1.046 \\
4 \\
(1.08 \\
83)\end{array}$ & $\begin{array}{c}1.041 \\
6 \\
(1.08 \\
33)\end{array}$ & $\begin{array}{c}1.038 \\
8 \\
(1.08 \\
04)\end{array}$ & $\begin{array}{c}1.038 \\
0 \\
(1.07 \\
95)\end{array}$ & $\begin{array}{c}1.135 \\
4 \\
(1.18 \\
08)\end{array}$ & $\begin{array}{c}1.116 \\
8 \\
(1.16 \\
15)\end{array}$ & $\begin{array}{c}1.101 \\
7 \\
(1.14 \\
58)\end{array}$ & $\begin{array}{c}1.090 \\
5 \\
(1.13 \\
41)\end{array}$ & $\begin{array}{c}1.083 \\
1 \\
(1.12 \\
64)\end{array}$ & $\begin{array}{c}1.069 \\
8 \\
(1.11 \\
26)\end{array}$ & $\begin{array}{c}1.063 \\
(1.10 \\
55)\end{array}$ & $\begin{array}{c}1.057 \\
7 \\
(1.10 \\
00)\end{array}$ & $\begin{array}{c}1.054 \\
2 \\
(1.09 \\
64)\end{array}$ & $\begin{array}{c}1.052 \\
9 \\
(1.09 \\
50)\end{array}$ \\
\hline $\begin{array}{l}0 . \\
8\end{array}$ & $\begin{array}{c}1.09 \\
44 \\
(1.13 \\
82)\end{array}$ & $\begin{array}{c}1.082 \\
5 \\
(1.12 \\
58)\end{array}$ & $\begin{array}{c}1.073 \\
9 \\
(1.11 \\
69)\end{array}$ & $\begin{array}{c}1.069 \\
0 \\
(1.11 \\
18)\end{array}$ & $\begin{array}{c}1.067 \\
6 \\
(1.11 \\
03)\end{array}$ & $\begin{array}{c}1.240 \\
7 \\
(1.29 \\
03)\end{array}$ & $\begin{array}{c}1.207 \\
7 \\
(1.25 \\
60)\end{array}$ & $\begin{array}{c}1.180 \\
8 \\
(1.22 \\
80)\end{array}$ & $\begin{array}{c}1.160 \\
8 \\
(1.20 \\
72)\end{array}$ & $\begin{array}{c}1.147 \\
8 \\
(1.19 \\
37)\end{array}$ & $\begin{array}{c}1.124 \\
1 \\
(1.16 \\
91)\end{array}$ & $\begin{array}{c}1.112 \\
1 \\
(1.15 \\
66)\end{array}$ & $\begin{array}{c}1.102 \\
5 \\
(1.14 \\
66)\end{array}$ & $\begin{array}{c}1.096 \\
4 \\
(1.14 \\
03)\end{array}$ & $\begin{array}{c}1.094 \\
1 \\
(1.13 \\
79)\end{array}$ \\
\hline $\begin{array}{l}1 . \\
0\end{array}$ & $\begin{array}{c}1.14 \\
76 \\
(1.19 \\
35)\end{array}$ & $\begin{array}{c}1.128 \\
9 \\
(1.17 \\
41)\end{array}$ & $\begin{array}{c}1.115 \\
5 \\
(1.16 \\
01)\end{array}$ & $\begin{array}{c}1.107 \\
9 \\
(1.15 \\
22)\end{array}$ & $\begin{array}{c}1.105 \\
7 \\
(1.14 \\
99)\end{array}$ & $\begin{array}{c}1.376 \\
1 \\
(1.43 \\
11)\end{array}$ & $\begin{array}{c}1.324 \\
5 \\
(1.37 \\
75)\end{array}$ & $\begin{array}{c}1.282 \\
5 \\
(1.33 \\
38)\end{array}$ & $\begin{array}{c}1.251 \\
3 \\
(1.30 \\
14)\end{array}$ & $\begin{array}{c}1.231 \\
0 \\
(1.28 \\
02)\end{array}$ & $\begin{array}{c}1.193 \\
9 \\
(1.24 \\
17)\end{array}$ & $\begin{array}{c}1.175 \\
1 \\
(1.22 \\
21)\end{array}$ & $\begin{array}{c}1.160 \\
2 \\
(1.20 \\
66)\end{array}$ & $\begin{array}{c}1.150 \\
5 \\
(1.19 \\
65)\end{array}$ & $\begin{array}{c}1.147 \\
0 \\
(1.19 \\
29)\end{array}$ \\
\hline $\begin{array}{l}2 . \\
0\end{array}$ & $\begin{array}{c}1.59 \\
03 \\
(1.65 \\
39)\end{array}$ & $\begin{array}{c}1.515 \\
4 \\
(1.57 \\
60)\end{array}$ & $\begin{array}{c}1.462 \\
0 \\
(1.52 \\
05)\end{array}$ & $\begin{array}{c}1.431 \\
5 \\
(1.48 \\
88)\end{array}$ & $\begin{array}{c}1.422 \\
7 \\
(1.47 \\
96)\end{array}$ & $\begin{array}{c}2.504 \\
3 \\
(2.60 \\
45)\end{array}$ & $\begin{array}{c}2.297 \\
9 \\
(2.38 \\
98)\end{array}$ & $\begin{array}{c}2.129 \\
8 \\
(2.21 \\
50)\end{array}$ & $\begin{array}{c}2.005 \\
2 \\
(2.08 \\
54)\end{array}$ & $\begin{array}{c}1.924 \\
0 \\
(2.00 \\
10)\end{array}$ & $\begin{array}{c}1.775 \\
7 \\
(1.84 \\
67)\end{array}$ & $\begin{array}{c}1.700 \\
5 \\
(1.76 \\
85)\end{array}$ & $\begin{array}{c}1.640 \\
9 \\
(1.70 \\
65)\end{array}$ & $\begin{array}{c}1.602 \\
2 \\
(1.66 \\
63)\end{array}$ & $\begin{array}{c}1.587 \\
9 \\
(1.65 \\
14)\end{array}$ \\
\hline $\begin{array}{l}3 . \\
0\end{array}$ & $\begin{array}{c}2.32 \\
82 \\
(2.42 \\
13)\end{array}$ & $\begin{array}{c}2.159 \\
7 \\
(2.24 \\
61)\end{array}$ & $\begin{array}{c}2.039 \\
6 \\
(2.12 \\
12)\end{array}$ & $\begin{array}{c}1.970 \\
8 \\
(2.04 \\
96)\end{array}$ & $\begin{array}{c}1.951 \\
1 \\
(2.02 \\
91)\end{array}$ & $\begin{array}{c}4.384 \\
7 \\
(4.56 \\
01)\end{array}$ & $\begin{array}{c}3.920 \\
2 \\
(4.07 \\
70)\end{array}$ & $\begin{array}{c}3.542 \\
2 \\
(3.68 \\
39)\end{array}$ & $\begin{array}{c}3.261 \\
7 \\
(3.39 \\
22)\end{array}$ & $\begin{array}{c}3.079 \\
0 \\
(3.20 \\
22)\end{array}$ & $\begin{array}{c}2.745 \\
2 \\
(2.85 \\
50)\end{array}$ & $\begin{array}{c}2.576 \\
0 \\
(2.67 \\
90)\end{array}$ & $\begin{array}{c}2.442 \\
1 \\
(2.53 \\
98)\end{array}$ & $\begin{array}{c}2.354 \\
9 \\
(2.44 \\
91)\end{array}$ & $\begin{array}{c}2.322 \\
7 \\
(2.41 \\
56)\end{array}$ \\
\hline
\end{tabular}

values shown inside ( ) obtained by ANSYS values shown outside ( ) obtained by theoretical analysis

Table 12: indicating the Values $\left(\lambda_{N L T} / \lambda_{L T}\right)$ for stepped simply supported compound beam of $I_{12}=\mathbf{0 . 8}$

\begin{tabular}{|c|c|c|c|c|c|c|c|c|c|c|c|c|c|c|c|}
\hline \multicolumn{16}{|c|}{$\begin{array}{c}\frac{\lambda_{N L T}}{\lambda_{L T}} \\
\text { Compound stepped simply supported Beam } \\
I_{12=0.8} \\
\end{array}$} \\
\hline \multirow[b]{2}{*}{$a / r_{2}$} & \multicolumn{5}{|c|}{$\begin{aligned} & \text { Steel-aluminum } \\
E_{12}= & 0.34459, \alpha_{12}=1.9552\end{aligned}$} & \multicolumn{5}{|c|}{$\begin{array}{c}\text { Titanium-aluminum } \\
E_{12}=0.6182, \alpha_{12}=2.7871\end{array}$} & \multicolumn{5}{|c|}{$\begin{array}{c}\text { Copper-aluminum } \\
E_{12}=0.6375, \alpha_{12}=1.3367\end{array}$} \\
\hline & 0.1 & 0.2 & 0.3 & 0.4 & 0.5 & 0.1 & 0.2 & 0.3 & 0.4 & 0.5 & 0.1 & 0.2 & 0.3 & 0.4 & 0.5 \\
\hline 0.0 & $\begin{array}{c}1.00 \\
00\end{array}$ & $\begin{array}{c}1.00 \\
00\end{array}$ & $\begin{array}{c}1.00 \\
00\end{array}$ & $\begin{array}{c}1.00 \\
00\end{array}$ & $\begin{array}{c}1.00 \\
00\end{array}$ & $\begin{array}{c}1.00 \\
00\end{array}$ & $\begin{array}{c}1.00 \\
00\end{array}$ & $\begin{array}{c}1.00 \\
00\end{array}$ & $\begin{array}{c}1.00 \\
00\end{array}$ & $\begin{array}{c}1.00 \\
00\end{array}$ & $\begin{array}{c}1.00 \\
00\end{array}$ & $\begin{array}{c}1.00 \\
00\end{array}$ & $\begin{array}{c}1.00 \\
00\end{array}$ & $\begin{array}{c}1.00 \\
00\end{array}$ & 1.0000 \\
\hline 0.2 & $\begin{array}{c}1.00 \\
60 \\
(1.0 \\
462)\end{array}$ & $\begin{array}{c}1.00 \\
53 \\
(1.0 \\
455)\end{array}$ & $\begin{array}{c}1.00 \\
48 \\
(1.0 \\
450)\end{array}$ & $\begin{array}{c}1.00 \\
45 \\
(1.0 \\
447)\end{array}$ & $\begin{array}{c}1.00 \\
45 \\
(1.0 \\
446)\end{array}$ & $\begin{array}{c}1.01 \\
53 \\
(1.0 \\
559)\end{array}$ & $\begin{array}{c}1.01 \\
35 \\
(1.0 \\
540)\end{array}$ & $\begin{array}{c}1.01 \\
20 \\
(1.0 \\
525)\end{array}$ & $\begin{array}{c}1.01 \\
09 \\
(1.0 \\
513)\end{array}$ & $\begin{array}{c}1.01 \\
01 \\
(1.0 \\
505)\end{array}$ & $\begin{array}{c}1.00 \\
79 \\
(1.0 \\
482)\end{array}$ & $\begin{array}{c}1.00 \\
73 \\
(1.0 \\
476)\end{array}$ & $\begin{array}{c}1.00 \\
68 \\
(1.0 \\
471)\end{array}$ & $\begin{array}{c}1.00 \\
66 \\
(1.0 \\
469)\end{array}$ & $\begin{array}{c}1.0065 \\
(1.0468)\end{array}$ \\
\hline 0.4 & $\begin{array}{c}1.02 \\
39 \\
(1.0\end{array}$ & $\begin{array}{c}1.02 \\
11 \\
(1.0\end{array}$ & $\begin{array}{c}1.01 \\
91 \\
(1.0\end{array}$ & $\begin{array}{c}1.01 \\
80 \\
(1.0\end{array}$ & $\begin{array}{c}1.01 \\
79 \\
(1.0\end{array}$ & $\begin{array}{c}1.06 \\
13 \\
(1.1\end{array}$ & $\begin{array}{c}1.05 \\
41 \\
(1.0\end{array}$ & $\begin{array}{c}1.04 \\
81 \\
(1.0 \\
\end{array}$ & $\begin{array}{c}1.04 \\
37 \\
(1.0\end{array}$ & $\begin{array}{c}1.04 \\
07 \\
(1.0\end{array}$ & $\begin{array}{c}1.03 \\
16 \\
(1.0\end{array}$ & $\begin{array}{c}1.02 \\
92 \\
(1.0\end{array}$ & $\begin{array}{c}1.02 \\
74 \\
(1.0\end{array}$ & $\begin{array}{c}1.02 \\
62 \\
(1.0\end{array}$ & $\begin{array}{c}1.0260 \\
(1.0670)\end{array}$ \\
\hline
\end{tabular}


Post-buckling analysis of a simply supported compound beams made of two symmetrically distributed

\begin{tabular}{|c|c|c|c|c|c|c|c|c|c|c|c|c|c|c|c|}
\hline & $649)$ & $619)$ & $599)$ & $587)$ & $586)$ & $038)$ & $963)$ & $900)$ & $854)$ & $823)$ & $729)$ & $704)$ & $685)$ & $672)$ & \\
\hline \multirow{5}{*}{0.6} & 1.05 & 1.04 & 1.04 & 1.04 & 1.04 & 1.13 & 1.12 & 1.10 & 1.09 & 1.09 & 1.07 & 1.06 & 1.06 & 1.05 & \\
& 37 & 74 & 29 & 05 & 02 & 80 & 17 & 83 & 83 & 16 & 12 & 58 & 16 & 90 & 1.0584 \\
& $(1.0$ & $(1.0$ & $(1.0$ & $(1.0$ & $(1.0$ & $(1.1$ & $(1.1$ & $(1.1$ & $(1.1$ & $(1.1$ & $(1.1$ & $(1.1$ & $(1.1$ & $(1.1$ & $(1.1007)$ \\
& $958)$ & $893)$ & $846)$ & $821)$ & $818)$ & $835)$ & $666)$ & $526)$ & $422)$ & $353)$ & $14)$ & $084)$ & $041)$ & $014)$ & \\
\hline \multirow{5}{*}{0.8} & 1.09 & 1.08 & 1.07 & 1.07 & 1.07 & 1.24 & 1.21 & 1.19 & 1.17 & 1.16 & 1.12 & 1.11 & 1.10 & 1.10 & \\
& 55 & 43 & 63 & 20 & 14 & 54 & 64 & 26 & 48 & 29 & 66 & 69 & 94 & 49 & 1.1039 \\
& $(1.1$ & $(1.1$ & $(1.1$ & $(1.1$ & $(1.1$ & $(1.2$ & $(1.2$ & $(1.2$ & $(1.2$ & $(1.2$ & $(1.1$ & $(1.1$ & $(1.1$ & $(1.1$ & $(1.1481)$ \\
& $393)$ & $277)$ & $194)$ & $149)$ & $143)$ & $952)$ & $651)$ & $403)$ & $218)$ & $094)$ & $717)$ & $616)$ & $538)$ & $491)$ & \\
\hline \multirow{6}{*}{1.0} & 1.14 & 1.13 & 1.11 & 1.11 & 1.11 & 1.38 & 1.33 & 1.30 & 1.27 & 1.25 & 1.19 & 1.18 & 1.17 & 1.16 & \\
& 92 & 17 & 93 & 25 & 16 & 34 & 81 & 09 & 31 & 46 & 78 & 27 & 10 & 39 & 1.1623 \\
& $(1.1$ & $(1.1$ & $(1.1$ & $(1.1$ & $(1.1$ & $(1.4$ & $(1.3$ & $(1.3$ & $(1.3$ & $(1.3$ & $(1.2$ & $(1.2$ & $(1.2$ & $(1.2$ & $(1.2088)$ \\
& $952)$ & $770)$ & $641)$ & $570)$ & $561)$ & $387)$ & $916)$ & $529)$ & $240)$ & $048)$ & $457)$ & $300)$ & $178)$ & $105)$ & \\
\hline \multirow{5}{*}{2.0} & 1.59 & 1.52 & 1.47 & 1.45 & 1.44 & 2.53 & 2.35 & 2.20 & 2.09 & 2.01 & 1.79 & 1.73 & 1.68 & 1.65 & \\
& 67 & 68 & 70 & 00 & 65 & 35 & 23 & 36 & 23 & 86 & 12 & 07 & 39 & 56 & 1.6493 \\
& $(1.6$ & $(1.5$ & $(1.5$ & $(1.5$ & $(1.5$ & $(2.6$ & $(2.4$ & $(2.2$ & $(2.1$ & $(2.0$ & $(1.8$ & $(1.7$ & $(1.7$ & $(1.7$ & $(1.7153)$ \\
& $606)$ & $879)$ & $361)$ & $080)$ & $044)$ & $348)$ & $464)$ & $917)$ & $760)$ & $993)$ & $628)$ & $999)$ & $513)$ & $218)$ & \\
\hline \multirow{6}{*}{3.0} & 2.34 & 2.18 & 2.07 & 2.01 & 2.00 & 4.45 & 4.04 & 3.70 & 3.45 & 3.29 & 2.78 & 2.64 & 2.53 & 2.47 & \\
& 26 & 54 & 33 & 24 & 46 & 04 & 27 & 81 & 77 & 20 & 01 & 40 & 88 & 52 & 2.4609 \\
& $(2.4$ & $(2.2$ & $(2.1$ & $(2.0$ & $(2.0$ & $(4.6$ & $(4.2$ & $(3.8$ & $(3.5$ & $(3.4$ & $(2.8$ & $(2.7$ & $(2.6$ & $(2.5$ & $(2.5593)$ \\
& $363)$ & $728)$ & $562)$ & $929)$ & $848)$ & $284)$ & $044)$ & $564)$ & $960)$ & $237)$ & $913)$ & $498)$ & $404)$ & $742)$ & \\
\hline
\end{tabular}

values shown inside () obtained by ANSYS

values shown outside () obtained by theoretical analysis 